\title{
Coexistence of superconductivity and incommensurate magnetic order
}

\author{
Andrzej Ptok, Maciej M. Maśka, and Marcin Mierzejewski \\ Institute of Physics, University of Silesia, 40-007 Katowice, Poland
}

\begin{abstract}
The influence of incommensurate spin density waves (SDW) on superconductivity in unconventional superconductors is studied by means of the Bogolubov-de Gennes (BdG) equations. Exploiting translational symmetries of a magnetically ordered two-dimensional system we propose an approach that allows to solve the BdG equations on much larger clusters than it is usually possible for inhomogeneous systems. Applying this approach we demonstrate that the presence of incommensurate spin density waves induces real-space inhomogeneity of the superconducting order parameter even in the absence of external magnetic field. In this case a homogeneous order parameter of the Bardeen-Cooper-Schrieffer-type superconducting state is slightly modulated, or equivalently, a small fraction of the charge carriers form Cooper pairs with non-zero total momentum. However, when a sufficiently strong magnetic field is applied, the homogeneous component of the order parameter is suppressed and the system transits to the Fulde-Ferrell-Larkin-Ovchinnikov (FFLO) state, where the order parameter oscillates changing sign. We show that for $s$-wave pairing the presence of external magnetic field diminishes the destructive influence of the SDW order on superconductivity. A simple explanation of this effect is also proposed.
\end{abstract}

PACS numbers: 74.25.Ha, 74.70.Tx

\section{INTRODUCTION}

The interplay of magnetism and superconductivity has been investigated both theoretically and experimentally for many decades. In 1957 Ginzburg demonstrated that superconductivity and long range ferromagnetic order compete with each other making their coexistence almost impossible $\underline{\underline{1}}$ However, it turned out that this incompatibility can be overcome by spatial separation as it takes place, e.g., in some Chevrel phases. The situation is particularly interesting in the case of unconventional superconductors, where the superconducting phase is often close to a magnetic one and, what is even more important, magnetic and superconducting phases are formed by the same electrons. Among others, unconventional superconductors include cuprates, heavy fermion systems $\Sigma^{2-4}$ and the recently discovered iron pnictides,$\underline{\underline{5}-\underline{7}}$ A microscopic description of the interplay between magnetism and superconductivity still represents a complex and unsolved problem.

There are, however, unconventional superconductors with coexisting magnetic and superconducting orders. Such a coexistence is observed, e.g., in iron pnictides ${ }^{8}$ and in heavy fermion $\mathrm{CeRhIn}_{5} \stackrel{4}{*}$ In these systems crossovers from a purely magnetic phase to a phase with coexisting orders and then to a purely superconducting phase (or in the opposite direction, depending on the control parameter) are observed. These examples show that magnetism can coexist with superconductivity, but they do not say much about the mutual interplay of these orders, i.e., whether they coexist because of a cooperation or despite a competition. The competition can be deduced indirectly from the phase diagrams (see e.g., Ref. 4) where it shows up as a negative correlation between the magnetic and superconducting transition temperatures. The onset of magnetic order in the vicinity of superconducting vortex cores $^{\underline{9}-11}$ gives additional sup- port for this competition. Some additional light can be shed on this problem by studying a system similar to $\mathrm{CeRhIn}_{5}$ but with cobalt instead of rhodium. $\mathrm{CeCoIn}_{5}$ is unusual in that the magnetic order occurs only inside the boundaries of the superconducting phase and vanishes together with superconductivity at the upper critical field.$^{2}$ This indicates that in $\mathrm{CeCoIn}_{5}$ there is a mutual cooperation between superconducting and magnetic orders. The physical origin of the high-field and low-temperature (HFLT) phase with coexisting orders remains controversial, especially with respect to its relation to the FFLO superconductivity $\underline{12-19}$ While the early results on $\mathrm{CeCoIn}_{5}$ were interpreted mostly in terms of the FFLO scenario $\stackrel{20-24}{\underline{2}}$ the discovery of the incommensurate SDW in the HFLT regime $2,3,25-27$ raised the question whether this phase is of magnetic or FFLO nature. Contradictory conclusions can be found even in the very recent theoretical28-38 and experimental papers $2,3,18,19,39-43$ The observed field independence of the wave-vector associated with SDW has been considered as a key argument against the FFLO scenario ${ }^{30}$

The FFLO state is characterized by the formation of the Cooper pairs with nonzero total momentum $\boldsymbol{q}$ and the spatially inhomogeneous order parameter (OP) of the Fulde-Ferrell ${ }^{12} \Delta(\boldsymbol{r}) \sim \exp (i \boldsymbol{r} \cdot \boldsymbol{q})$ or the LarkinOvchinnikov $\underline{13} \Delta(\boldsymbol{r}) \sim \cos (\boldsymbol{r} \cdot \boldsymbol{q})$ types. Usually, there are more than two equivalent $\boldsymbol{q}$-vectors which give the same upper critical field for the FFLO state, while the lowest free energy is obtained for OP being a linear combination of several plain waves $\underline{4-51}$ with a complicated spatial modulation of the OP. Since any additional modulation of the OP in the real space requires an additional components in the momentum space, inclusion of several momenta $\boldsymbol{q}$ should be necessary in the presence of mechanisms which break/modify the translational invariance, like impurities $33,52,53$ or incommensurate SDW.

The spatial structure of the FFLO OP has been de- 
termined mostly from the solution of the BdG equations in the real space $49,51-53$ Such an approach is best suited for investigations of the FFLO phase in the presence of impurities or vortices when the translational invariance is broken. However, for a coexisting SDW and FFLO phases calculations in the momentum space allow one to benefit from a possible translational invariance in the direction perpendicular to the SDW modulation. In this paper we develop such an approach and study the influence of SDW on $s$ and $d$-wave superconductivity. By carrying out numerical calculations on systems up to $10^{5}$ sites we show that incommensurate SDW itself favors pairing with nonzero momentum for both the symmetries. What is interesting, in the presence of incommensurate SDW, the $s$-wave superconductivity persists up to much higher magnetic fields than in systems without SDW. We present simple arguments explaining this effect.

\section{MODEL AND APPROACH}

While the spatial structure of the magnetic order in HFLT phase has been determined from several experiments, $2,25,26,41$ the spatial structure of the superconducting OP remains unknown. Therefore, the experimental data on SDW will be taken as a phenomenological input in our calculations. Solving the BdG equations for the superconducting OP we determine how the assumed SDW affects formation of Cooper pairs with various total momenta. We investigate the following Hamiltonian on a two-dimensional square lattice

$$
H=H_{0}+H_{s(d)},
$$

where $H_{s}\left(H_{d}\right)$ represents pairing interaction responsible for $s$-wave ( $d$-wave) superconductivity and

$$
H_{0}=-t \sum_{\langle i, j\rangle, \sigma} c_{i \sigma}^{\dagger} c_{j \sigma}-\sum_{i, \sigma}\left\{\sigma\left[h+M\left(\boldsymbol{R}_{i}\right)\right]+\mu\right\} c_{i \sigma}^{\dagger} c_{i \sigma} .
$$

Here, $c_{i \sigma}^{\dagger}$ creates an electron with spin $\sigma$ at site $i, t$ is the hopping integral between the nearest-neighbor sites and $\mu$ is the chemical potential. We focus on the role of the external magnetic field $h$ and incommensurate SDW order $M\left(\boldsymbol{R}_{i}\right)=M_{0} \cos \left(\boldsymbol{R}_{i} \cdot \boldsymbol{Q}_{\mathrm{SDW}}\right)$. Following Refs. [2, 25, 26, and 41] we take either $\boldsymbol{Q}_{\mathrm{SDW}}=(Q, Q)$ or $\boldsymbol{Q}_{\mathrm{SDW}}=(Q, \pi)$. Hamiltonian (2) includes the Zeeman pair breaking but it neglects the orbital effects of magnetic field, which are the most effective pair-breaking mechanism in many superconductors. Here, however, we focus on heavy fermion systems where this mechanism is ineffective due to the large electron effective mass. This mechanism does not play a role also in layered systems provided the field is applied parallel to the planes.

The mean-field form of the on-site pairing interaction for $s$-wave superconductivity reads

$$
H_{s}=\sum_{i}\left(\Delta_{i} c_{i \uparrow}^{\dagger} c_{i \downarrow}^{\dagger}+\text { H.c. }-\frac{\left|\Delta_{i}\right|^{2}}{V_{s}}\right),
$$

with $\Delta_{i}=V_{s}\left\langle c_{i \downarrow} c_{i \uparrow}\right\rangle$ and $V_{s}<0$. In the case of inter-site pairing we assume

$$
H_{d}=\sum_{i, \alpha}\left[\frac{\Delta_{i}^{\alpha}}{2}\left(c_{i \uparrow}^{\dagger} c_{i+\alpha \downarrow}^{\dagger}-c_{i \downarrow}^{\dagger} c_{i+\alpha \uparrow}^{\dagger}\right)+\text { H.c. }-\frac{\left|\Delta_{i}^{\alpha}\right|^{2}}{V_{d}}\right],
$$

where $\Delta_{i}^{\alpha}=\frac{V_{d}}{2}\left\langle c_{i+\alpha \downarrow} c_{i \uparrow}-c_{i+\alpha \uparrow} c_{i \downarrow}\right\rangle$ for $\alpha \in\{\hat{x}, \hat{y}\}$ and $V_{d}<0$. This form of the interaction Hamiltonian allows for an arbitrary inter-site singlet pairing (e.g., the extended $s$-wave). However, the experimental results suggest $d$-wave superconductivity and we restrict further analysis to this type of pairing. Representing the superconducting order parameters by their Fourier transforms

$$
\Delta_{i}^{(\alpha)}=\sum_{\boldsymbol{q}} \Delta_{\boldsymbol{q}}^{(\alpha)} \exp \left(i \boldsymbol{q} \cdot \boldsymbol{R}_{i}\right)
$$

one obtains the Hamiltonian in the momentum space

$$
\begin{aligned}
H_{0}= & \sum_{\boldsymbol{k}, \sigma} \mathcal{E}_{\boldsymbol{k} \sigma} c_{\boldsymbol{k} \sigma}^{\dagger} c_{\boldsymbol{k} \sigma} \\
- & \sum_{\boldsymbol{k}, \sigma} \frac{\sigma M_{0}}{2}\left(c_{\boldsymbol{k} \sigma}^{\dagger} c_{\boldsymbol{k}-\boldsymbol{Q}_{\mathrm{SDW}}, \sigma}+c_{\boldsymbol{k} \sigma}^{\dagger} c_{\boldsymbol{k}+\boldsymbol{Q}_{\mathrm{SDW}}, \sigma}\right), \\
H_{s}= & \sum_{\boldsymbol{k} \boldsymbol{q}} \Delta_{\boldsymbol{q}} c_{\boldsymbol{k} \uparrow}^{\dagger} c_{-\boldsymbol{k}+\boldsymbol{q} \downarrow}^{\dagger}+\text { H.c. }-\frac{N}{V_{s}} \sum_{\boldsymbol{q}}\left|\Delta_{\boldsymbol{q}}\right|^{2}, \\
H_{d}= & \sum_{\boldsymbol{k q}} \sum_{\alpha} \Delta_{\boldsymbol{q}}^{\alpha} d_{\alpha}(\boldsymbol{k}, \boldsymbol{q}) c_{\boldsymbol{k} \uparrow}^{\dagger} c_{-\boldsymbol{k}+\boldsymbol{q} \downarrow}^{\dagger}+\text { H.c. } \\
& -\frac{N}{V_{d}} \sum_{\boldsymbol{q}, \alpha}\left|\Delta_{\boldsymbol{q}}^{\alpha}\right|^{2},
\end{aligned}
$$

where $\mathcal{E}_{\boldsymbol{k} \sigma}=-2 t\left(\cos k_{x}+\cos k_{y}\right)-\mu-\sigma h, d_{x(y)}(\boldsymbol{k}, \boldsymbol{q})=$ $\cos \left[k_{x(y)}-q_{x(y)} / 2\right]$ and

$$
\begin{aligned}
\Delta_{\boldsymbol{q}} & =\frac{V_{s}}{N} \sum_{\boldsymbol{k}}\left\langle c_{-\boldsymbol{k}+\boldsymbol{q} \downarrow} c_{\boldsymbol{k} \uparrow}\right\rangle, \\
\Delta_{\boldsymbol{q}}^{x(y)} & =\frac{V_{d}}{N} \sum_{\boldsymbol{k}} d_{x(y)}(\boldsymbol{k}, \boldsymbol{q})\left\langle c_{-\boldsymbol{k}+\boldsymbol{q} \downarrow} c_{\boldsymbol{k} \uparrow}\right\rangle .
\end{aligned}
$$

The resulting Hamiltonian can be diagonalized by the transformation

$$
c_{\boldsymbol{k} \sigma}=\sum_{n}\left(u_{\boldsymbol{k} n \sigma} \gamma_{n \sigma}-\sigma v_{\boldsymbol{k} n \sigma}^{*} \gamma_{n \bar{\sigma}}^{\dagger}\right)
$$

where $\gamma_{n \sigma}$ are the quasiparticle operators while $u_{\boldsymbol{k} n \sigma}$ and $v_{\boldsymbol{k} n \sigma}$ fulfil the BdG equations

$$
\sum_{\boldsymbol{p}}\left(\begin{array}{cc}
H_{\boldsymbol{k p} \sigma} & \tilde{\Delta}_{\boldsymbol{k p}} \\
\tilde{\Delta}_{\boldsymbol{k} \boldsymbol{p}}^{*} & -H_{\boldsymbol{k} \boldsymbol{\overline { \sigma }}}^{*}
\end{array}\right)\left(\begin{array}{c}
u_{\boldsymbol{p} n \sigma} \\
v_{\boldsymbol{p} n \bar{\sigma}}
\end{array}\right)=E_{n \sigma}\left(\begin{array}{c}
u_{\boldsymbol{k} n \sigma} \\
v_{\boldsymbol{k} n \bar{\sigma}}
\end{array}\right)
$$

Here,

$$
H_{\boldsymbol{k p} \sigma}=\delta_{\boldsymbol{k} \boldsymbol{p}} \mathcal{E}_{\boldsymbol{k} \sigma}-\delta_{\boldsymbol{k}, \boldsymbol{p}-\boldsymbol{Q}_{\mathrm{SDW}}} \frac{\sigma M_{0}}{2}-\delta_{\boldsymbol{k}, \boldsymbol{p}+\boldsymbol{Q}_{\mathrm{SDW}}} \frac{\sigma M_{0}}{2},
$$

is the normal state Hamiltonian and

$$
\tilde{\Delta}_{\boldsymbol{k} \boldsymbol{p}}= \begin{cases}\sum_{\boldsymbol{q}} \Delta_{\boldsymbol{q}} \delta_{\boldsymbol{k},-\boldsymbol{p}+\boldsymbol{q}} & \text { for } s \text {-wave } \\ \sum_{\boldsymbol{q}} \Delta_{\boldsymbol{q}}^{\alpha} d_{\alpha}(\boldsymbol{k}, \boldsymbol{q}) \delta_{\boldsymbol{k},-\boldsymbol{p}+\boldsymbol{q}} & \text { for } d \text {-wave. }\end{cases}
$$


Superconducting order parameters are determined selfconsistently from Eqs. (910) together with

$$
\begin{aligned}
\left\langle c_{-\boldsymbol{k}+\boldsymbol{q} \downarrow} c_{\boldsymbol{k} \uparrow}\right\rangle & =\sum_{n} v_{-\boldsymbol{k}+\boldsymbol{q}, n \downarrow}^{*} u_{\boldsymbol{k} n \uparrow} f\left(E_{n \uparrow}\right) \\
& -\sum_{n} u_{-\boldsymbol{k}+\boldsymbol{q}, n \downarrow} v_{\boldsymbol{k} n \uparrow}^{*} f\left(-E_{n \downarrow}\right),
\end{aligned}
$$

where $f(E)=[1+\exp (\beta E)]^{-1}$ is the Fermi-Dirac distribution function. In particular, we focus on the relation between the BCS $\Delta_{\boldsymbol{q}=(0,0)}^{(x, y)}$ and FFLO $\Delta_{\boldsymbol{q} \neq(0,0)}^{(x, y)}$ components of the superconducting OP.

An unrestricted search for solutions of the BdG equations in the momentum space has no advantage over the standard analysis in the real space. However, the advantage becomes evident when the system is invariant under translations along one particular axis. Such a case will be considered in the present work, where we assume that the direction of FFLO modulation $\boldsymbol{q}$ is parallel to incommensuration wave-vector of the magnetic order $\boldsymbol{\delta}=(\pi, \pi)-\boldsymbol{Q}_{\mathrm{SDW}}$. Under this assumption Eq. (12) represents eigenproblem of a block matrix. For the $L \times L$ system with $\boldsymbol{\delta} \| \hat{\boldsymbol{d}}$ where $\hat{\boldsymbol{d}}$ is a diagonal unit vector $\hat{\boldsymbol{d}}=(1,1)$, the blocks are not larger than $2 L \times 2 L$ and each block account for particles and holes which momenta are connected by the following relation: $\boldsymbol{p}^{\prime}=\boldsymbol{p}+2 \pi \hat{\boldsymbol{d}} n / L$, where $n=0, \ldots, L-1$. In the second case, when $\boldsymbol{\delta} \| \hat{\boldsymbol{x}}$ with $\hat{\boldsymbol{x}}=(1,0)$, the biggest blocks consist of $4 L \times 4 L$ elements. Within each block we consider particles and holes with momenta connected via $\boldsymbol{p}^{\prime}=\boldsymbol{p}+2 \pi \hat{\boldsymbol{x}} n / L$, as well as $\boldsymbol{p}^{\prime}=\boldsymbol{p}+(0, \pi)+2 \pi \hat{\boldsymbol{x}} n / L$, where $n=0, \ldots, L-1$.

Certainly, we cannot exclude a possibility that an additional modulation in the perpendicular direction may lead to a further lowering of the free energy and stabilization of the superconducting phase beyond the boundaries obtained in the present studies.

\section{RESULTS AND DISCUSSION}

In the numerical calculations we take $\mu=-0.2 t$, what gives the occupation number slightly below one electron per lattice site. The magnitudes of the pairing potentials are $V_{s}=-2.0 t$ for $s$-wave and $V_{d}=-1.2 t$ for $d$-wave pairing. The BdG equations have been solved for clusters up to $256 \times 256$ sites at temperature $k_{B} T=10^{-4} t$.

The physically relevant solutions of the BdG equations are determined according to the following procedure: we start from a small system, e.g., $64 \times 64$ and for each magnetic field $h$ and SDW amplitude $M_{0}$ we iteratively solve the $\mathrm{BdG}$ equations starting from LO states with all possible momenta $\boldsymbol{q}$. Depending on $\boldsymbol{Q}_{\mathrm{SDW}}$ these momenta are either along $(1,1)$ or $(1,0)$ directions. Then, the solution with the lowest free energy together with the LO states with neighboring momenta are taken as the initial states in iterative solution of the BdG equations on much larger clusters, e.g., $256 \times 256$. Although our approach to the inter-site pairing remains valid for an arbitrary singlet state, we solve the BdG equations starting from an initial $d$-wave state with $\Delta_{i}^{x}=-\Delta_{i}^{y}$.

Solving the BdG equations in the absence of SDW, we have found for the assumed dispersion relation and the Fermi energy that the FFLO state with vectors $\boldsymbol{q}$ along $(1,0)$ direction have lower free energy than the states with $\boldsymbol{q}$ along $(1,1)$. Therefore, we will show results only for SDW with the incommensuration wave-vector $\boldsymbol{\delta} \|(1,0)$. However, we have found the same qualitative results also for $\boldsymbol{\delta} \|(1,1)$ and $\boldsymbol{q} \|(1,1)$, when the free energy is only slightly higher. Note that we assume that the onset of incommensurate SDW does not change the direction of modulation of $\Delta_{i}$. It is the only restriction imposed on the solutions of the BdG equations in our approach. Further on, when discussing the $d$-wave superconductivity we present site-dependent superconducting order parameter defined for site $i$ as an average of $\Delta_{i j}$ over four bonds connecting site $i$ to its neighbors:

$$
\Delta_{i}=\frac{1}{4}\left(\Delta_{i, i+\hat{x}}+\Delta_{i, i-\hat{x}}-\Delta_{i, i+\hat{y}}-\Delta_{i, i-\hat{y}}\right)
$$
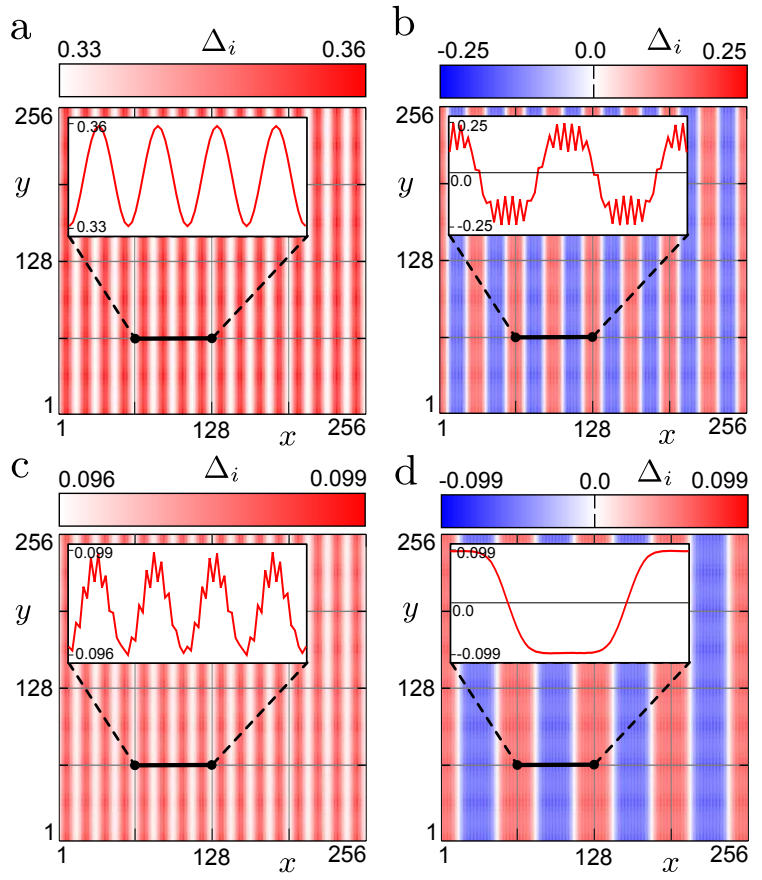

FIG. 1. (Color online) Real-space profile of the superconducting OP for $\boldsymbol{Q}_{\mathrm{SDW}}=\left(\frac{15}{16}, 1\right) \pi$ calculated on a $256 \times 256$ cluster. Panels a and b show $s$-wave superconductivity with $M_{0}=0.2 t$ for $h=0$ and $h=0.25 t$, respectively. Panels c and d show the $d$-wave case with $M_{0}=0.1 t$ and $h=0$ (panel c) and $h=0.25 t$ (panel d).

Fig. 10 shows the real-space profiles of the superconducting OP for $s$-wave (upper panels) and $d$-wave (lower panels) superconductivity in the absence (left column) as well as in the presence (right column) of the magnetic field. One can see that for $h=0, \Delta_{i}$ is spatially modulated with periodicity given by the incommensuration $\boldsymbol{\delta}$. In other words, considering translationally invariant 
BCS state in the presence of incommensurate SDW is an approximation and leads to a state with the free energy higher than that of the inhomogeneous states shown in Figs. 17a and 17. From Eqs. (9, 10) one immediately finds that incommensurate SDW itself induces Cooper pairs with momenta along $\boldsymbol{\delta}$. The magnitude of SDW is expected to determine whether these components dominate or, as in the case shown in Fig. 1, they represent corrections to the BCS superconductivity.

Similarly to the case of non-magnetic systems, external field favors pairing with a non-zero momentum also in the presence of SDW. As demonstrated in the right panels in Fig. 1, for sufficiently strong field, the BCS component $\Delta_{q=0}$ vanishes and the superconducting OP changes sign in the real-space. However, the spatial profile of $\Delta_{i}$ is very different from a standard cosine dependence. Contrary to the LO phase, several components with different momenta of Cooper pairs give significant contribution to the superconducting OP.
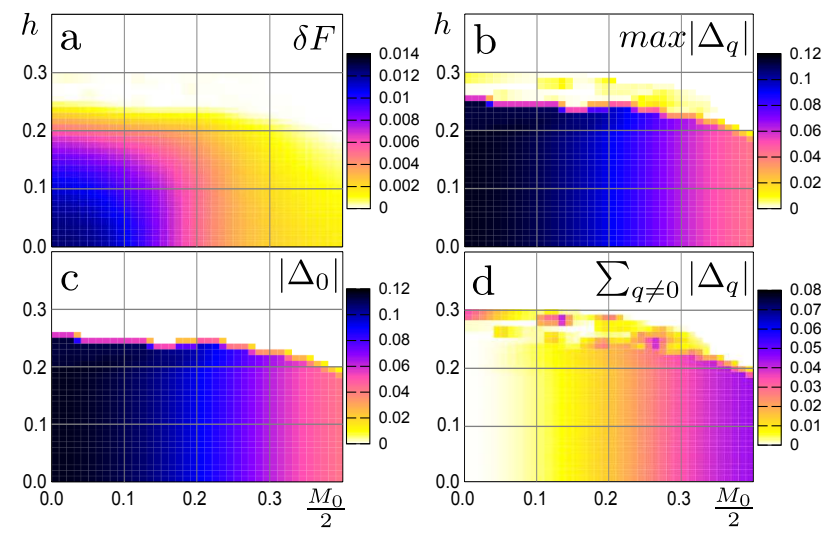

FIG. 2. (Color online) Phase diagrams for $d$-wave superconductivity with $Q_{\mathrm{SDW}}=\left(\frac{15}{16}, 1\right) \pi$ obtained on a $64 \times 64$ clusters. Panel a shows the free energy $F$ relative to the normal-state energy $F_{N}$ under the same conditions $\delta F=F_{N}-F$. Panels $\mathrm{b}$ and $\mathrm{c}$ show the maximal $\max \left|\Delta_{q}^{x}\right|$ and the BCS $\left|\Delta_{0}^{x}\right|$ components of the superconducting $\mathrm{OP}$, respectively, while panel d shows sum of all amplitudes with nonzero momentum of Cooper pairs $\sum_{q \neq 0}\left|\Delta_{q}^{x}\right|$.

Results shown in Fig. 11indicate that incommensurate SDW affects the total momentum of Cooper pairs both in the presence and in the absence of external magnetic field. Then, the key question is whether these results are generic for incommensurate SDW or, in contrary, they require a fine-tuning of $M_{0}$ and $h$. In order to answer this question we have calculated phase diagrams in the $M_{0}-h$ plane. The diagrams shown in Figs. 2] and 3 are the main results of our study. In order to determine the properties of the superconducting state (especially the role of Cooper pairs with nonzero momentum) we have calculated the following quantities: $\delta F=F_{N}-F$, where $F$ and $F_{N}$ denote the free energy and the free energy of a nonsuperconducting (normal) state, respectively; $\max \left|\Delta_{q}\right|-$ maximal single components of OP [see Eqs. (910)]; $\left|\Delta_{0}\right|=\left|\Delta_{q=0}\right|$ - the BCS component of OP,

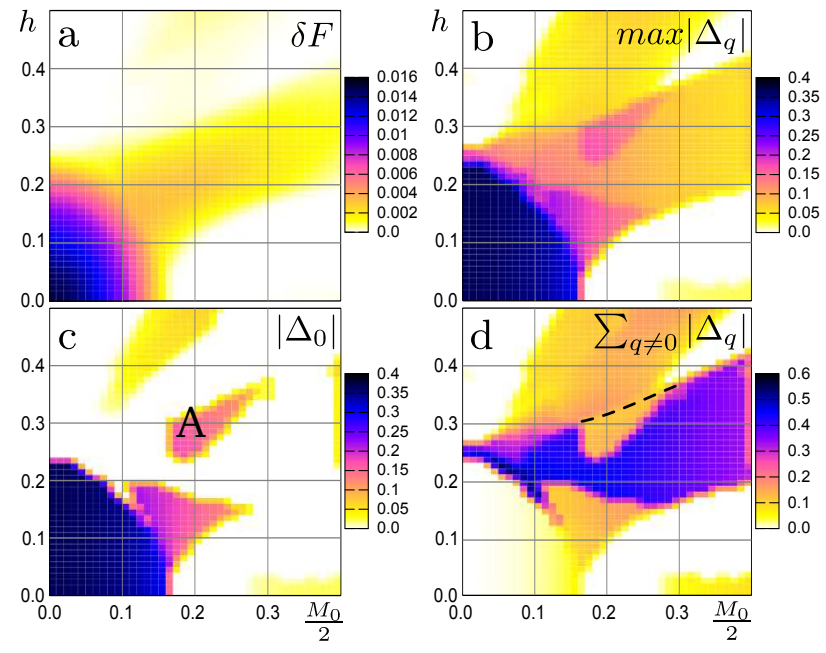

FIG. 3. (Color online) The same as in Fig. 2 but for $s$-wave symmetry $\left(\Delta_{q}^{x} \rightarrow \Delta_{q}\right)$.

and $\sum_{\boldsymbol{q} \neq 0}\left|\Delta_{\boldsymbol{q}}\right|$ - sum of all amplitudes with nonzero momentum of Cooper pairs. Increasing the magnitude of incommensurate SDW causes reduction of $\left|\Delta_{0}\right|$ accompanied by an increase of $\sum_{\boldsymbol{q} \neq 0}\left|\Delta_{\boldsymbol{q}}\right|$. This holds true for both symmetries. It is a clear indications that incommensurate SDW acts in detriment of BCS superconductivity and favors pairing with nonzero momentum of Cooper pairs. Since also $\max \left|\Delta_{\boldsymbol{q}}\right|$ decreases when $M_{0}$ increases, this superconducting state is very different from the standard LO superconductivity, when only two components with opposite momenta are relevant.

A clear distinction between $d$-wave (Fig. 2) and $s$ wave (Fig. 3 ) superconductivity shows up in the phase diagrams when superconductivity is simultaneously affected by SDW and external magnetic field $h$. In the case of $d$-wave superconductivity there is no unusual interplay between these two mechanism and the role SDW is rather negligible as long as $M_{0}$ is smaller than the critical field. This stands in strong contrast to the results for $s$-wave superconductivity. When considered separately, the external field and SDW are strong pairbreaking mechanisms. However, they are not so destructive upon superconductivity when they emerge together. As we consider a real-space pairing with rather strong pairing potentials, our approach should be applicable to extremely type-II superconductors. Hence, it should be possible to explain the obtained results investigating the spatial variation of a local effective field defined by $h_{\mathrm{eff}}\left(\boldsymbol{R}_{i}\right) \equiv h+M_{0} \cos \left(\boldsymbol{Q}_{\mathrm{SDW}} \cdot \boldsymbol{R}_{i}\right)$ on a very short lengthscale of the order of the coherence length. Let us define a fraction of sites

$$
\Psi^{s}=\frac{1}{N} \sum_{i} \theta\left(h_{c}-\left|h_{\mathrm{eff}}\left(\boldsymbol{R}_{i}\right)\right|\right)
$$


and bonds

$$
\Psi^{d}=\frac{1}{4 N} \sum_{\langle i, j\rangle} \theta\left(h_{c}-\left|h_{\mathrm{eff}}\left(\boldsymbol{R}_{i}\right)\right|\right) \theta\left(h_{c}-\left|h_{\mathrm{eff}}\left(\boldsymbol{R}_{j}\right)\right|\right),
$$

where the effective field $h_{\text {eff }}$ is smaller than the critical field $h_{c}$ determined in the absence of SDW. Here, $\theta(\ldots)$ is the Heaviside step function. These quantities are shown in Fig. 4. One can see that at least in the case of $s$-wave superconductivity $\Psi^{s}$ provides very simple explanation of the general structure of the phase diagram shown in Fig. 3. In the presence of incommensurate SDW, external magnetic field $h$ proportional to $M_{0}$ increases the number of lattice sites where the effective magnetic field is smaller than $h_{c}$ and in this way it effectively screens superconductivity against SDW. Of course, at the same time it increases the effective field $h_{\text {eff }}$ at some other sites, but in the case of the FFLO superconductivity the order parameter can be adjusted in such a way that the influence of these sites is minimized.
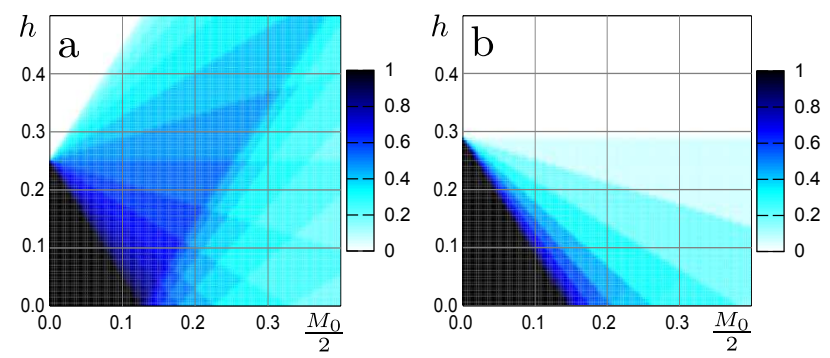

FIG. 4. (Color online) Fraction of sites (a) and bonds (b), where the magnitude of the effective magnetic field is lower than $h_{c}=0.25 t$ (a) and $h_{c}=0.29 t$ (b). Results are obtained on a $128 \times 128$ lattice for $Q_{\mathrm{SDW}}=\left(\frac{15}{16}, 1\right) \pi$.

In summary, we have solved the BdG equations for su- perconductivity coexisting with (assumed) incommensurate SDW. We have considered a case when spatial modulations of the superconducting and antiferromagnetic orders takes place in the same direction. The translational invariance in the perpendicular directions allowed us to study systems as large as $256 \times 256$. Our numerical data provide a clear evidence that incommensurate SDW itself diminishes the role of the BCS pairing and simultaneously favors formation of Cooper pairs with nonzero total-momentum. It has recently been demonstrated that also a complementary dependence, where tendency toward formation of incommensurate SDW is enhanced by the presence of the FFLO order, is possible $\underline{\underline{34}}$ These observations hold true for $s$-wave and $d$-wave superconductivity and supports the hypothesis that FFLO-type of superconductivity exist in the HFLT phase of heavyfermion superconductor $\mathrm{CeCoIn}_{5}$.

For $s$-wave superconductivity we have found rather surprising result concerning the case when superconductivity is affected simultaneously by external magnetic field and the incommensurate SDW. We have demonstrated that these two mechanisms are less destructive upon $s$-wave superconductivity when they emerge together. It means that under external magnetic field superconductivity may coexists with stronger SDW than in the absence of magnetic field.

\section{ACKNOWLEDGMENTS}

The authors acknowledge support under Grant No. N N202 052940 from Ministry of Science and Higher Education (Poland). A.P. acknowledges support from the UPGOW project, cofinanced by the European Social Fund.
1 V. L. Ginzburg, Sov. Phys. JETP 4, 153 (1957)

2 M. Kenzelmann, T. Strässle, C. Niedermayer, M. Sigrist, B Padmanabhan, M Zolliker, A. D. Bianchi, R. Movshovich, E. D. Bauer, J. L. Sarrao, J. D. Thompson, Science 321, 1652 (2008).

3 M. Kenzelmann, S. Gerber, N. Egetenmeyer, J. L. Gavilano, Th. Strssle, A. D. Bianchi, E. Ressouche, R. Movshovich, E. D. Bauer, J. L. Sarrao, and J. D. Thompson, Phys. Rev. Lett. 104, 127001 (2010).

4 T. Park, F. Ronning, H. Q. Yuan, M. B. Salamon, R. Movshovich, J. L. Sarrao and J. D. Thompson, Nature 440, 65 (2006).

${ }^{5}$ R. H. Liu, G. Wu, T. Wu, D. F. Fang, H. Chen, S. Y. Li, K. Liu, Y. L. Xie, X. F. Wang, R. L. Yang, L. Ding, C. He, D. L. Feng, and X. H. Chen, Phys. Rev. Lett. 101, 087001 (2008).

6 R. M. Fernandes and J. Schmalian, Phys. Rev. B 82, 014521 (2010).
7 A. B. Vorontsov, M. G. Vavilov, and A. V. Chubukov, Phys. Rev. B 79, 060508(R) (2009)

8 A. J. Drew, C. Niedermeyer, P. J. Baker, F. L. Pratt, S. J. Blundell, T. Lancaster, R. H. Liu, G. Wu, X. H. Chen, I. Watanabe, V. K. Malik, A. Dubroka, M. Rs̈sle, K. W. Kim, C. Baines and C. Bernhard, Nature Materials 8, 310 (2009).

9 B. Lake, H.M. Ronnow, N.B. Christensen, G. Aeppli, K. Lefmann, D.F. McMorrow, P. Vorderwisch, P. Smeibidl, N. Mangkorntong, T. Sasagawa, M. Nohra, H. Takagi, and T.E. Mason, Nature (London) 415, 299 (2002).

10 Y. Chen, Z. D. Wang, J.-X. Zhu, and C. S. Ting, Phys. Rev. Lett. 89, 217001 (2002).

11 M. M. Maśka and M. Mierzejewski, Phys. Rev. B 68, 024513 (2003).

12 P. Fulde and R. A. Ferrel, Phys. Rev. 135, A550 (1964).

13 A. I. Larkin and Yu. N. Ovchinnikov, Zh. Eksp. Teor. Fiz. 47, 1136 (1964) [Sov. Phys. JETP 20, 762 (1965)]. 
${ }^{14}$ Y. Matsuda and H. Shimahara, J. Phys. Soc. Jpn. 76, 051005 (2007).

15 C. Capan, A. Bianchi, R. Movshovich, A. D. Christianson, A. Malinowski, M. F. Hundley, A. Lacerda, P. G. Pagliuso, and J. L. Sarrao, Phys. Rev. B 70, 134513 (2004).

16 P. Javorský, E. Colineau, F. Wastin, F. Jutier, J.-C. Griveau, P. Boulet, R. Jardin, and J. Rebizant, Phys. Rev. B 75, 184501 (2007).

17 R. Okazaki, H. Shishido, T. Shibauchi, M. Konczykowski, A. Buzdin, and Y. Matsuda, Phys. Rev. B 76, 224529 (2007).

18 M. Nicklas, C. F. Miclea, J. L. Sarrao, J. D. Thompson, G. Sparn and F. Steglich, J. Low Temp. Phys. 146, 669 (2007)

19 R. R. Urbano, M. J. Graf, N. J. Curro, and B. L. Young, J. Low Temp. Phys. 158, 635 (2010).

20 A. Bianchi, R. Movshovich, C. Capan, P. G. Pagliuso, and J. L. Sarrao, Phys. Rev. Lett. 91, 187004 (2003).

${ }^{21}$ H. A. Radovan, N. A. Fortune, T. P. Murphy, S. T. Hannahs, E. C. Palm, S. W. Tozer, and D. Hall, Nature 425, 51 (2003).

22 R. Movshovich, M. Jaime, J. D. Thompson, C. Petrovic, Z. Fisk, P. G. Pagliuso, and J. L. Sarrao, Phys. Rev. Lett. 86, 5152 (2001).

23 D. Hall, E. C. Palm, T. P. Murphy, S. W. Tozer, Z. Fisk, U. Alver, R. G. Goodrich, J. L. Sarrao, P. G. Pagliuso, and T. Ebihara, Phys. Rev. B 64, 212508 (2001).

24 A. Bianchi, R. Movshovich, N. Oeschler, P. Gegenwart, F. Steglich, J. D. Thompson, P. G. Pagliuso, and J. L. Sarrao, Phys. Rev. Lett. 89, 137002 (2002).

25 B.-L. Young, R. R. Urbano, N. J. Curro, J. D. Thompson, J. L. Sarrao, A. B. Vorontsov, and M. J. Graf, Phys. Rev. Lett. 98, 036402 (2007).

26 G. Koutroulakis, V. F. Mitrovic, M. Horvatic, C. Berthier, G. Lapertot, and J. Flouquet, Phys. Rev. Lett. 101, 047004 (2008).

27 K. Kumagai, N. Kondoh, H. Shishido, and Y. Matsuda, Physica C 470, S533 (2010)

28 A. Aperis, G. Varelogiannis, P. B. Littlewood and B. D. Simons, J. Phys.: Condens. Matter 20, 434235 (2008)

29 A. Aperis, G. Varelogiannis, P. B. Littlewood, and B.D. Simons, J. Supercond. Nov. Magn. 22, 115 (2009)

30 A. Aperis, G. Varelogiannis, and P. B. Littlewood, Phys. Rev. Lett. 104, 216403 (2010).

31 K. Miyake, J. Phys. Soc. Jpn. 77, 123703 (2008).

${ }^{32}$ R. Ikeda, Y. Hatakeyama, and K. Aoyama, Phys. Rev. B 82, 060510(R) (2010).

33 R. Ikeda, Phys. Rev. B 81, 060510(R) (2010).

${ }^{34}$ M. Mierzejewski, A. Ptok and M. M. Maśka, Phys. Rev. B 80, $174525(2009)$
35 M. M. Maśka, M. Mierzejewski, J. Kaczmarczyk, and J. Spałek, Phys. Rev. B 82, 054509 (2010).

36 J. Kaczmarczyk and J. Spałek, Phys. Rev. B 79, 214519 (2009).

37 G. Zwicknagl, J. Wosnitza, International Journal of Modern Physics B 24, 3915 (2010).

38 D. F. Agterberg, M. Sigrist, and H. Tsunetsugu, Phys. Rev. Lett. 102, 207004 (2009).

39 Y. Tokiwa, R. Movshovich, F. Ronning, E. D. Bauer, A. D. Bianchi, Z. Fisk, and J. D. Thompson, Phys. Rev. B 82, 220502(R) (2010).

40 J. Spehling, R. H. Heffner, J. E. Sonier, N. Curro, C. H. Wang, B. Hitti, G. Morris, E. D. Bauer, J. L. Sarrao, F. J. Litterst, and H.-H. Klauss, Phys. Rev. Lett. 103, 237003 (2009).

41 G. Koutroulakis, M. D. Stewart, Jr., V. F. Mitrović, M. Horvatić, C. Berthier, G. Lapertot, and J. Flouquet, Phys. Rev. Lett. 104, 087001 (2010).

42 G. Knebel, D. Aoki, J. P. Brison, L. Howald, G. Lapertot, J. Panarin, S. Raymond, and J. Flouquet, Physica Status Solidi B 247, 557 (2010)

43 E. Blackburn, P. Das, M. R. Eskildsen, E. M. Forgan, M. Laver, C. Niedermayer, C. Petrovic, and J. S. White, Phys. Rev. Lett. 105, 187001 (2010).

44 H. Shimahara, J. Phys. Soc. Jpn. 67, 736 (1998).

45 J. A. Bowers and K. Rajagopal, Phys. Rev. D 66, 065002 (2002).

46 C. Mora and R. Combescot, Europhys. Lett. 66, 833 (2004).

47 R. Combescot and G. Tonini, Phys. Rev. B 72, 094513 (2005).

48 C. Mora and R. Combescot: Phys. Rev. B 71, 214504 (2005).

49 Q. Wang, H.-Y. Chen, C.-R. Hu, and C. S. Ting, Phys. Rev. Lett. 96, 117006 (2006).

50 P. Dey, S. Basu and R Kishore, J. Phys.: Condens. Matter 21, 355602 (2009).

51 Y. Yanase and M. Sigrist, J. Phys. Cond Mat. 23, 094219 (2011); Y. Yanase, Phys. Rev. B 80, 220510(R) (2009); Y. Yanase and M. Sigrist, J. Phys. Soc. Jpn. 78, 114715 (2009).

52 A. Ptok, Acta Phys. Pol. A 118, 420 (2010).

53 Q. Wang, C.-R. Hu, and C.-S. Ting , Phys. Rev. B 74, 212501 (2006); Q. Wang, C.-R. Hu, and C.-S. Ting, Phys. Rev. B 75, 184515 (2007).

54 A. Ptok, M. M. Maśka, and M. Mierzejewski, J. Phys.: Condens. Matter 21, 295601 (2009). 